\title{
COVID-19 y educación a distancia digital: preconfinamiento, confinamiento y posconfinamiento
}

\section{(COVID-19 and digital distance education: pre- confinement, confinement and post-confinement)}

\author{
Lorenzo García Aretio \\ Universidad Nacional de Educación a Distancia, UNED (España)
}

DOI: http://dx.doi.org/10.5944/ried.24.1.28080

\section{Cómo referenciar este artículo:}

García Aretio, L. (2021). COVID-19 y educación a distancia digital: preconfinamiento, confinamiento y posconfinamiento. RIED. Revista Iberoamericana de Educación a Distancia, 24(1), pp. 09-32. doi: http://dx.doi. org/10.5944/ried.24.1.28080

\section{Resumen}

La pandemia COVID-19 ha venido generando cambios y disrupciones en amplios sectores de la actividad humana. La educación ha sido uno de los más afectados debido a la imposición administrativa del cierre total de los centros educativos en gran parte de los países del mundo. La modalidad de educación a distancia, fundamentalmente en soporte digital, vino a ofrecer soluciones de emergencia a dicha crisis. En este trabajo se lleva a cabo una reflexión sobre las percepciones previas al COVID-19 relativas a esta modalidad educativa. Posteriormente se aportan ideas sobre la forma en que se acometió mayoritariamente la respuesta educativa al confinamiento a través de una educación de emergencia en remoto que se alejaba considerablemente de lo apropiado en diseños y desarrollos de una educación a distancia de calidad. Fueron muchos los errores cometidos y, por tanto, demasiadas las percepciones negativas por parte de muchos estudiantes, familias y docentes, bastantes de estos últimos ya reacios previamente a estos formatos más novedosos. Finalmente se aborda en el artículo la problemática más reciente, relativa a los tiempos de posconfinamiento, en los que no se prevé que en los centros presenciales todos los estudiantes puedan acudir a las aulas físicas en el mismo espacio y tiempo. Se ofrecen sugerencias sobre cómo abordar esta problemática a través de soluciones de hibridación, de una enseñanza y aprendizaje mixtos, combinados o, mejor, integrados y flexibles.

Palabras clave: COVID-19; educación a distancia; confinamiento; e-Learning; blendedlearning; aprendizaje híbrido; combinado; mixto; flexible. 


\begin{abstract}
The COVID-19 pandemic has brought changes and disruptions in wide areas of human activity. Education has been one of the most affected due to the administrative imposition of the total closure of educational centres in most of the countries of the world. The modality of distance education, fundamentally in digital support, was able to offer emergency solutions to this crisis. In this paper, a reflection is carried out on the prior perceptions to COVID-19, related to this educational modality. Subsequently, ideas are provided on the way in which the educational response to confinement was mainly undertaken, through remote emergency education that considerably deviated from what was appropriate in designs and developments of quality distance education. Many mistakes were made and, therefore, there were too many negative perceptions from many students, families and teachers, quite a few of the latter, previously reluctant to these latest formats. Finally, the article addresses the most recent problem, related to post-confinement times, in which it is not foreseen that every student can go to physical classrooms in face-to-face centres in the same space and time. Suggestions are offered on how to address this problem, through hybridization solutions, mixed teaching and learning, combined or, better, integrated and flexible.
\end{abstract}

Keywords: COVID-19; distance education; confinement; e-Learning; blended-learning; hybrid learning; blended; mixed; flexible.

Jamás en la historia se produjo un cierre universal de instalaciones educativas presenciales como el sucedido con motivo de la pandemia provocada por el COVID-19. Según datos actualizados de la $\mathrm{UNESCO}^{1}$, gobiernos de casi 200 países decretaron el cierre total o parcial de centros educativos. Y esa relación de países no paró de aumentar. Según la organización internacional, cerca de 1600 millones de niños, adolescentes y jóvenes se han visto afectados a nivel mundial por esta circunstancia, 91\% del total (figura 1), al igual que más de 60 millones de docentes abocados a un cambio radical y abrupto (IESALC-UNESCO, 2020). De ahí que UNESCO alentó a los diferentes gobiernos, y continúa haciéndolo, al uso de sistemas de educación a distancia, aprovechando las posibilidades que hoy ofrecen las tecnologías digitales. Son muchas, y con opciones muy diferentes, las plataformas y aplicaciones, algunas de ellas gratuitas, que permiten el estudio y la interacción con materiales y docentes. 
Figura 1. Alumnos afectados por la pandemia COVID-19

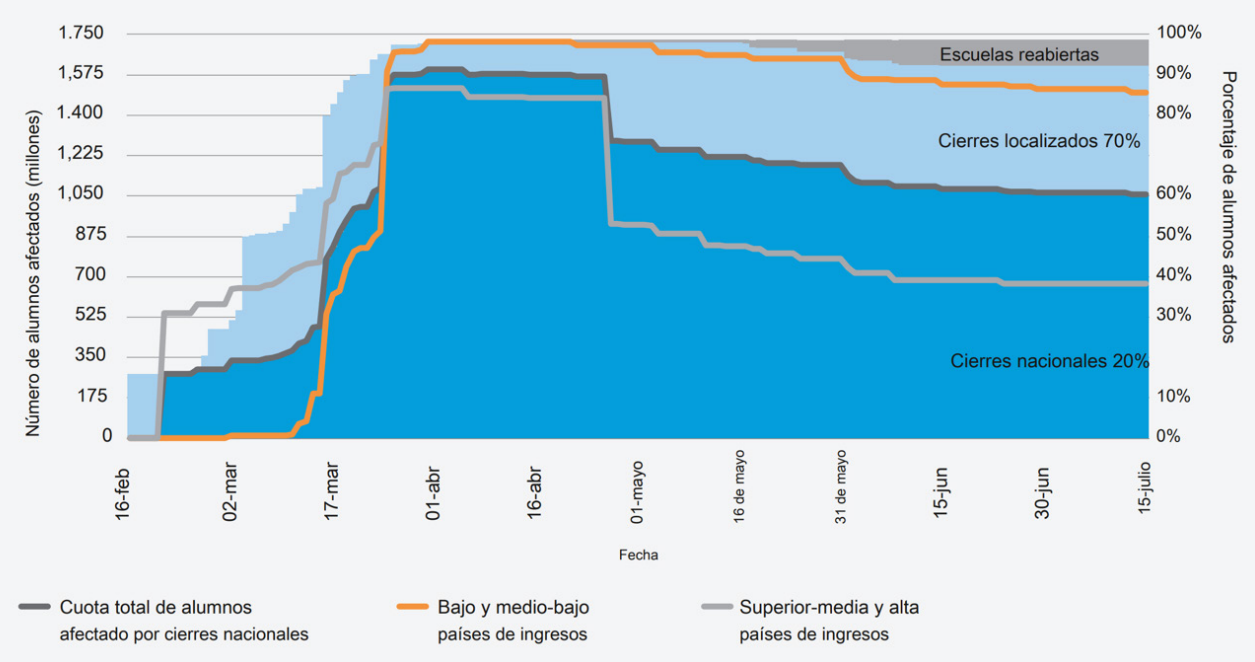

Fuente: https://en.unesco.org/covid19/educationresponse

Según la ONU (UN, 2020a), los cierres de los espacios educativos y de aprendizaje han afectado al 94\% de la población estudiantil mundial. Problema más acentuado aún en los países con escasos recursos. Las brechas de acceso se han incrementado con motivo de la pandemia, al reducir posibilidades a masas de estudiantes de poblaciones vulnerables o ya vulneradas. Esta crisis puede llevar a las poblaciones más pobres a una pérdida de aprendizaje irrecuperable, empujar al abandono de muchos estudiantes o a la dificultad para reiniciar las tareas escolares futuras debido, muy previsiblemente, a dificultades económicas generadas por la crisis. En concreto, las proyecciones de la ONU apuntan a que casi 24 millones de estudiantes de todos los niveles educativos podrían abandonar los estudios debido a dificultades económicas producidas por la pandemia.

Pero, sin duda, esta pandemia ha alimentado a espíritus innovadores que han ayudado a la búsqueda de soluciones educativas en época de confinamiento. La pandemia se ha convertido en un catalizador para que las instituciones educativas de todo el mundo busquen soluciones innovadoras en un período de tiempo relativamente corto (World Economic Forum, 2020).

Muchos de esos millones de alumnos, privados de la asistencia a la formación presencial en sus habituales centros de estudios, han mantenido, están manteniendo desde sus hogares un nivel de trabajo que pretende acercarse al anterior a la epidemia, con una gran exigencia en cuanto al esfuerzo y rigor exigidos digna de elogio. Clases emitidas en directo por internet, radio y televisión y, sobre todo, centenares 
de aplicaciones y programas informáticos que han ido creciendo en progresión geométrica para atender las necesidades educativas en todos los niveles del sistema.

Las operadoras de telecomunicaciones, las empresas y organizaciones privadas reaccionaron adecuadamente, ofreciendo, por una parte, la amplitud de banda necesaria, y por otra, servicios, programas, software, plataformas, etc., para facilitar la docencia en línea ante el momento de crisis sobrevenido sin avisar. Téngase en cuenta que existe una previsión de que el mercado mundial de la educación en línea llegue a una inversión global de 350.000 millones de dólares en 2025 (Research and Markets, 2019).

Paralelo a todo ello, los gobiernos tuvieron que actuar con reflejos facilitando en algunos países programas nacionales de educación a distancia mediante clases básicas que después podían ser complementadas por los docentes, también en línea, desde los diferentes centros o desde sus domicilios. Así, los estudiantes, desde sus hogares, podían seguir sus estudios reduciendo al mínimo las posibles pérdidas curriculares. Las respuestas de los diferentes países, ha sido desigual pero siempre orientada, obviamente, hacia metodologías no presenciales (World Bank, 2020).

Una interesante encuesta, 14th Annual Learning Tools Survey, 2020 (Hart, 2020), llevada a cabo después de decretarse el confinamiento y cierre de centros educativos, y publicada el pasado primero de septiembre, ofrece una interesante visión sobre cómo se está habilitando y apoyando el aprendizaje en estos tiempos en herramientas web (figura 2). 
Figura 2. Principales 100 herramientas Web utilizadas en 2020.

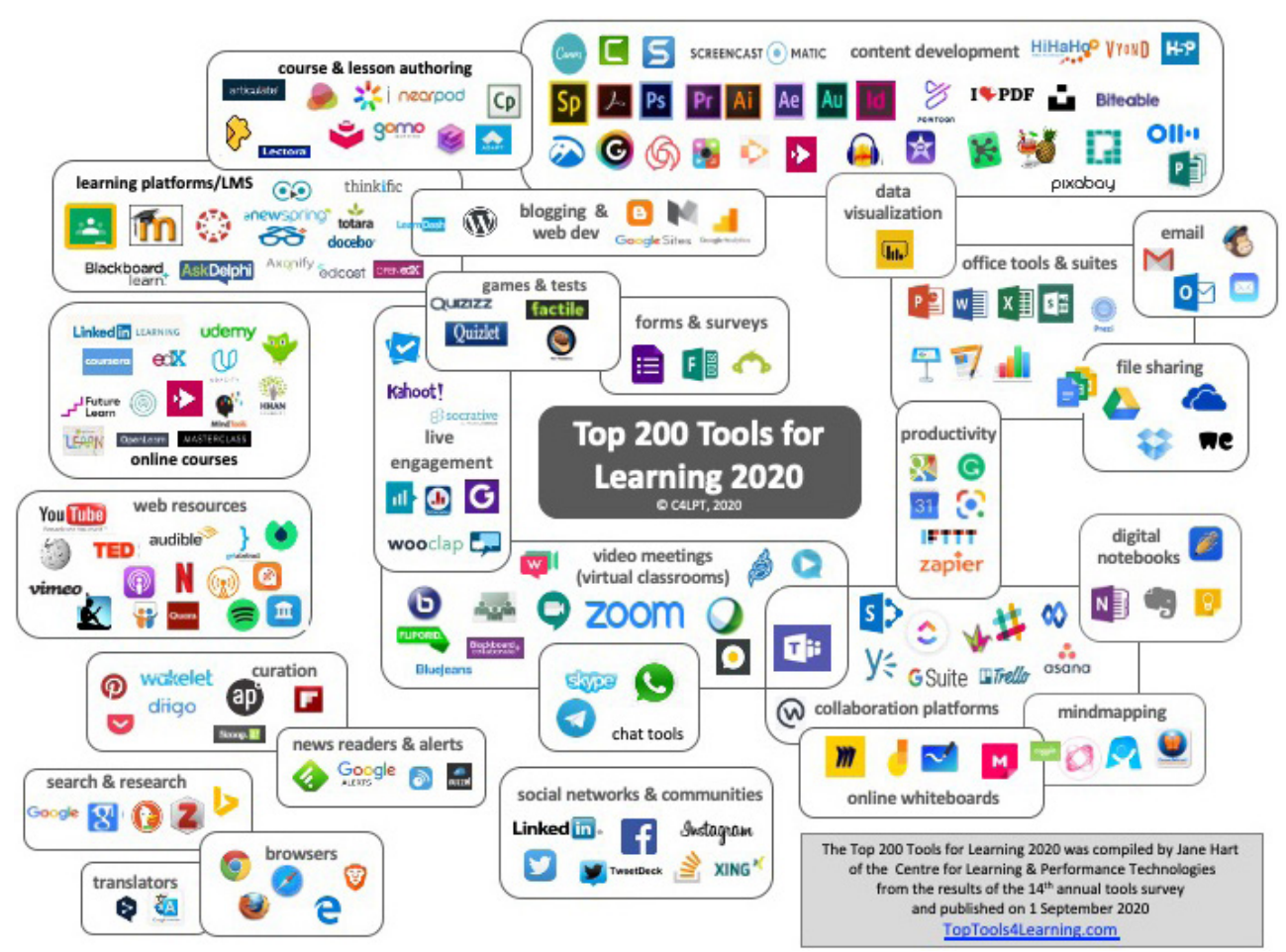

Fuente: https://www.toptools4learning.com/analysis-2020/

Sin duda, las universidades, contexto prioritario al que va dirigida esta revista, lo tuvieron y lo tienen más fácil. En primer lugar, porque muchas de ellas ya contaban con plataformas digitales que venían utilizando en algunas fases del desarrollo de su docencia, generalmente presencial. Y, en segundo lugar, porque la edad de los estudiantes hace más viable una enseñanza/aprendizaje de este corte. Lo más fácil en estos casos, para los docentes menos experimentados, ha sido la emisión en directo de una sesión idéntica al tiempo presencial anterior, o la grabación de esa sesión para su posterior recepción por parte de los estudiantes y que, en ambos casos, hacen las veces en remoto de las secuencias presenciales en el aula. Otro profesorado, más preparado en este tipo de estrategias metodológicas, trató de aprovechar las inmensas posibilidades de las plataformas digitales y de las propias redes sociales para desarrollar su tarea online de forma más creativa y eficaz.

Cierto que en estos tiempos ha sucedido que, ante el obligado cambio brusco en las formas de enseñar, de aprender y de evaluar, que no podían seguir siendo presenciales, una educación a distancia puesta muy en cuestión en tiempos preCOVID, 
tuvo que implementarse de urgencia, de forma masiva y con grandes limitaciones pedagógicas en tiempos de COVID, y se pretende aprovechar en formatos híbridos, combinados o de blended en épocas posteriores, posCOVID. Pues bien, en torno a estas tres fases pretende girar este artículo.

\section{PRECONFINAMIENTO Y EDUCACIÓN A DISTANCIA}

En torno a un $15 \%$ de los estudiantes universitarios españoles venían siguiendo estudios a distancia, sea total o parcialmente. Podría ser un porcentaje medio en los países desarrollados. Son, por tanto, millones de alumnos que estudiaron a distancia porque era su única alternativa o porque optaron por ese sistema frente a la oferta de estudio presencial.

No es ahora el momento de volver sobre la contrastada calidad de los estudios a distancia, una vez que éstos cuentan con diseños pedagógicos rigurosos, y docentes bien capacitados. La literatura es abundante con respecto a los fundamentos, posibilidades, perspectivas, retos y futuro de la educación a distancia digital. Esta revista RIED es buen ejemplo de lo que se señala. Son multitud de estudios recogidos en ella que dan sobrada muestra de esas posibilidades a las que se hace alusión. Y revistas como RIED hay más y trabajos científicos e informes internacionales publicados por organismos, asociaciones, gobiernos y grupos de investigación, son muchos y diversos, que llegan a conclusiones favorables respecto a la calidad de los aprendizajes y retención de la información en los sistemas digitales de enseñanza y aprendizaje.

En este trabajo priorizamos el concepto de "educación a distancia digital" como abarcador de un modelo de educación no presencial, soportado íntegramente en sistemas digitales. Se estaría hablando de una educación virtual, una enseñanza, un aprendizaje en línea, soportado en tecnologías, en la red, en Internet, en la web, e-learning, aprendizaje distribuido, etc. Todas estas denominaciones, como emergentes en su momento y sucesoras del original de educación a distancia, tienden a la apertura, a la no dependencia de ubicación física, a la flexibilidad de tiempo, espacio y ritmo de aprender, al aprendizaje activo, a la interacción (síncrona y asíncrona), etc., como elementos sustanciales en cualquier definición de estos conceptos (Singh y Thurman, 2019).

Sin embargo, al aproximarnos a la previsión del diseño de los estudios en época de confinamiento, surgen muchas voces exigiendo la vuelta $100 \%$ a la presencialidad por exclusivos motivos, según ellos, de la escasa calidad de la formación de los estudiantes, por falta de credibilidad de los sistemas a distancia o por su consideración de educación de segunda categoría. Esas voces la vienen aceptando sólo como respuesta de solución al confinamiento, pero provisional, indeseada y, a veces, despreciada.

Ha resultado frecuente que quienes atacaron a la educación a distancia en algunos de sus puntos débiles o en algunas de sus prácticas o presentaciones 
concretas de evidente escasa calidad, generalmente lo hicieron frente a una buena educación presencial, a una concreta y determinada educación presencial, fuerte en esos puntos. Pero no compararon fortalezas evidentes de las buenas prácticas en educación a distancia digital y la contrastaron con debilidades de una determinada presencial, o con prácticas presenciales poco aconsejables, que muestran escasos ejemplos de esas virtudes que se asignan a este formato. Nunca escribieron sobre la despersonalización de esa educación presencial impartida en aulas masificadas frente a las soluciones que podría aportar a esas masas de estudiantes una educación a distancia de calidad, de corte digital.

Algunos afirman que la educación virtual no puede erigirse en el nuevo paradigma educativo. Cierto que no debe intentar sustituir a la enseñanza presencial de calidad, pero sí ofrecer otra alternativa, otro paradigma diferente, con sus propias singularidades y valores. Se dice que Internet transmite datos, pero no valores. Dependerá de los usos y selección que se haga de esos datos para transformarlos en información y, a través de la guía del docente, elevarlos a conocimientos, actitudes y valores. ¿̇O es que la palabra hueca y monocorde de un mal docente presencial en un aula configura valores educativos superiores que los que pueda generar un excelente vídeo y unas actividades de aprendizaje ligadas a ese recurso?

Se dice que no es viable un aprendizaje sólido que no cuente con la cercanía afectiva entre docentes y alumnos, insisten, el docente debe armar vínculos emocionales que refuercen los meramente cognitivos. En efecto. Que esos vínculos generados en formatos educativos presenciales pueden conformarse como sólidos y rápidos, no se duda. Pero, igualmente, se configuran lazos afectivos, emocionales, a través de la red, aunque hay que saber manejarse en esos entornos para lograrlo. Es seguro que un buen docente a distancia consigue armar esos lazos de afecto antes y por encima de los conformados por un mal docente presencial en su aula.

Dicen quienes atacan estos formatos, que la docencia online se limita a un intercambio oral, escrito o con imágenes, no pudiendo disfrutarse del lenguaje no verbal. Quizás sea así en algunas prácticas y, por supuesto, podía serlo en la antigua educación a distancia. Se olvidó que existe la posibilidad de relación síncrona en línea a través de la cual puede mostrarse también ese lenguaje no verbal. Desde este trabajo, se insiste en que no deberíamos comparar al buen docente presencial con el mal docente a distancia, ni al revés.

Parece que se da un valor superlativo a la coincidencia de docente y estudiante en un lugar material y temporal para que pueda producirse un trabajo relacional, colaborativo, colectivo, etc. Probablemente quienes aseguran eso no han trabajado con grupos colaborativos en educación a distancia digital. Además de que puede trabajarse de forma síncrona, y a cualquier hora, puede hacerse en asincronía. Y los resultados parecen incuestionables.

Todo ello sin detenernos en lo que está viniendo, ligado a la inteligencia artificial y realidad virtual. Avance que va a permitir, por ejemplo, que un grupo de alumnos aprendan juntos, trayendo todas las ventajas del aula presencial al entorno virtual, 
aunque realmente se encuentren a miles de kilómetros unos de otros. Los docentes pueden entrar o no en esa aula virtual, observar las conductas de sus estudiantes, intervenir para orientar, etc. A estos avances de la realidad virtual podrá unirse en un próximo futuro el reconocimiento facial de los participantes en el grupo. Las expresiones faciales, como las que se observan en entornos presenciales, pueden mostrarnos el grado de interés, su estado de ánimo, en diferentes instancias de su participación en el grupo y así posibilitar reacciones adecuadas por parte de los educadores.

También estas tecnologías podrán reconocer y analizar la voz. Las formas de hablar importan para mejor entender lo que se dice y cómo se dice. Puede resultar de interés para los docentes en sus intervenciones puntuales. Llegarán también las posibilidades de ofrecer datos biométricos mientras se aprende, tales como la frecuencia cardiaca, la temperatura corporal, presión arterial, actividad cerebral, etc. Conocer cómo un estudiante se enfrenta al estrés, los niveles de esfuerzo requeridos ante determinadas tareas, etc. (Lozano, Cooper y Soto, 2020). Todo ello unido al big data y a la analítica de aprendizaje (Vol.23-2 de RIED) nos depara un futuro insospechado y no tan lejano.

Dicho lo cual, jamás deberá ignorarse el inmenso valor de la relación presencial en los procesos educativos. Pero no debemos aprovechar esa realidad para infravalorar otras formas de enseñar, de formar, de aprender y de educar, sí, de educar, que ya probaron su calidad cuando se aplicaron diseños pedagógicos apropiados y se dispuso de los recursos necesarios y del personal docente capacitado para ello.

Y, en todo caso, estas reflexiones van dirigidas a los estudios universitarios. Ya damos por supuesto que el sistema no universitario ha de basarse fundamentalmente en la relación presencial, en la socialización, en el contacto directo con la realidad, con el entorno físico. Aunque, también en estos casos, parece que se ha descubierto el mucho valor y aporte que las tecnologías pueden ofrecer para enriquecer y mejorar los propios logros pedagógicos de una buena relación presencial. Y en tiempos de confinamiento, se deben aprovechar las enseñanzas de las buenas prácticas en enseñanza y aprendizaje digitales.

Imaginemos las diferentes situaciones reales que se muestran en la tabla 1, relativas a estos contextos: a) docencia presencial sin uso de tecnologías, b) docencia presencial con tecnologías, c) sistema híbrido o combinado, y d) educación a distancia digital $100 \%$. Por otra parte, imaginemos los tamaños del grupo de estudiantes: a) pocos estudiantes, b) aula normal, c) aula masificada, y d) grandes masas de estudiantes. En la tabla se señalan las previsiones en cuanto a la mayor o menor adecuación del sistema empleado, en función del tamaño del grupo. 
Tabla 1. Adecuación del sistema empleado, en función del tamaño del grupo

\begin{tabular}{|l|l|l|l|l|}
\hline \multicolumn{1}{|c|}{ Modelo } & \multicolumn{1}{c|}{$\begin{array}{c}\text { Presencial sin } \\
\text { TIC }\end{array}$} & $\begin{array}{c}\text { Presencial } \\
\text { con TIC }\end{array}$ & $\begin{array}{c}\text { Híbrido } \\
\text { Combinado }\end{array}$ & $\begin{array}{c}\text { A Distancia } \\
100 \%\end{array}$ \\
\hline $\begin{array}{l}\text { Pocos } \\
\text { alumnos }\end{array}$ & $=$ & + & $=$ & $=$ \\
\hline $\begin{array}{l}\text { Aula normal } \\
\text { Aula } \\
\text { masificada }\end{array}$ & - & + & + & $=$ \\
\hline Grupo masivo & - & + & + & + \\
\hline
\end{tabular}

Leyenda: $=$ Aceptable; - Peor; + Mejor

Pero es el eterno afán de enfrentar las buenas prácticas presenciales con las malas prácticas a distancia, prescindiendo de la calidad real de una y otra y, como se muestra en la tabla, ignorando el contexto. ¿Por qué no se intenta hacer al revés? Lo más lógico sería no enfrentar nada sino aprovechar las ventajas de un formato y otro porque algunas acciones educativas concretas, algunas metodologías, recursos, actividades, etc., pueden ser intercambiables y más apropiadas según qué circunstancias, al margen de la forma de entrega de la educación.

La realidad es que tanto las instituciones, docentes y estudiantes de la modalidad a distancia como los de la modalidad presencial, nos encontrábamos en nuestras respectivas zonas de confort, protegidos por nuestros hábitos, rutinas, conductas y patrones de pensamiento pedagógicos aceptados y asumidos en la comunidad. El peligro de esta zona de confort es que sea muy rígida, nos sintamos tan a gusto que quedemos inmóviles, sin arriesgarnos, sin crecer, sin crear, sin apostar por nuevos retos.

\section{CONFINAMIENTO}

Y llegó la pandemia, el confinamiento. Y entonces, poco se les sacó de su zona de confort a quienes ya convivían con realidades de educación a distancia digital. Sin embargo, en lo que respecta a quienes se vieron removidos por el tsunami del COVID-19, es decir, quienes enseñaban y aprendían en formatos presenciales, la salida de ese espacio confortable y seguro, en muchos casos les hizo perder el norte. En otros, se sobrepusieron y dieron una respuesta muy digna.

Un hecho real que ha supuesto el confinamiento respecto a los sistemas educativos ha sido el de demostrar que los cambios en educación son posibles, incluso cuando se trata de cambios absolutamente radicales. Pero no es el cambio anhelado, éste al que nos hemos enfrentado, un cambio obligado, totalmente disruptivo, que supuso pasar abruptamente de una docencia presencial a otra a distancia, sin tiempo para la adaptación. A pesar de la urgencia, emergencia e improvisación, habrá que valorar e investigar cuáles variables, factores o elementos de esa traumática puesta 
en escena son bien aprovechables para épocas de posconfinamiento (UN, 2020a). Lo que sucede es que en esas circunstancias en que se acometió el cambio, podrá afirmarse que estos estudios difícilmente pueden ser representativos de lo que hasta el momento ha venido suponiendo una educación a distancia digital de calidad.

Podrían ser de dudosa credibilidad algunas investigaciones que se vienen realizando o se realizarán respecto a una enseñanza íntegramente en línea y relativas al rendimiento estudiantil en el contexto del COVID-19, a los grados de satisfacción de los usuarios, a los índices de calidad de los procesos, etc. La necesidad de disponer de un día para otro de un curso o una asignatura en línea se contradice con el tiempo real necesario para hacerlo con una calidad mínima (Hodges et al., 2020). Aunque estuvo bien que esos cambios improvisados hayan supuesto una solución temporal a un problema de máxima urgencia.

Soluciones que fueron diferentes según zonas geográficas e instituciones y en función de los recursos existentes y de la preparación de los docentes. Así surgieron experiencias de educación a distancia convencional centrada en el material impreso, el correo y el teléfono; emisiones por radio o televisión y la utilización de sistemas digitales, plataformas virtuales, etc.

Según el citado documento de la ONU (UN, 2020a) en tiempos de confinamiento, en los países desarrollados la educación a distancia digital cubría entre el 80 y el 85\% de la población estudiantil, mientras que, en los países de ingresos bajos, la cobertura de sistemas de educación virtual no llegaría al 50\%. Según datos de UNESCO (2020), la mitad del número total de estudiantes, unos 826 millones, que se mantuvo fuera del aula por la pandemia COVID-19, no tenía acceso a una computadora en el hogar y el 43\% (706 millones) no tenía Internet en casa, en un momento en que el aprendizaje a distancia con base digital se utilizó para garantizar la continuidad educativa en la gran mayoría de países. Por ésta y otras razones, la brecha digital, y otras brechas, posiblemente se han agudizado (Dreesen et al., 2020), de ahí que la ONU apueste por un cambio generalizado en el mundo del trabajo hacia una digitalización más inclusiva y centrada en las personas (UN, 2020b). Quizás suponga este grave problema un aldabonazo a las políticas socioeducativas implementadas por los diferentes gobiernos.

Aunque aumentan los esfuerzos complementarios en este sentido, la apuesta por la conectividad y el acceso universal a Internet debería ser una meta próxima en el tiempo como respuesta de los gobiernos a esta crisis. Se trata del derecho inalienable y universal a la educación que debería conllevar en tiempos digitales, como decimos, el derecho a la conectividad, accesibilidad, y a la mínima dotación de dispositivo tecnológico para hacerla realidad. Sería el caso de sectores de la población que no cuentan con conectividad o con dispositivos adecuados, o suficientes en familias con varios hijos, para la recepción de los contenidos y la interacción con los docentes. La televisión en estos casos puede reducir este problema, y más aún la radio. Estos medios de comunicación están mucho más extendidos, son accesibles a la población y de una eficacia probada para configurar acciones educativas de calidad. En todo 
caso, el reto mundial debe estar en no dejar a nadie atrás, de acuerdo con lo señalado en los Objetivos de Desarrollo Sostenible (ODS) de la Agenda 2030 de Naciones Unidas.

En lo que respecta a las universidades, más allá de que muchas de ellas contaban en sus planes estratégicos con previsiones de futuro para la enseñanza online, la realidad es que muy pocas de ellas estaban realmente preparadas para implementar de urgencia un modelo educativo plenamente digitalizado (u-Multirank, 2020). En las apresuradas lecturas y conocimiento de la realidad y de la puesta en práctica de inaplazables y, en muchos casos, poco deliberados sistemas en línea, especialmente en universidades españolas y de América Latina, y también con la pionera experiencia COVID de las universidades chinas (Bao, 2020), podrían señalarse algunas pistas respecto a los más relevantes inconvenientes encontrados en estas prácticas educativas de emergencia y en remoto, que podrían servir con el fin de considerarlos, en caso de nuevos cierres totales o parciales de instalaciones educativas:

- Dificultad de llegar a los alumnos que no cuentan con conectividad o con dispositivos apropiados.

- Saturación de las redes y escaso ancho de banda, sobre todo en instituciones con grandes masas de estudiantes.

- Situaciones de deserción o desánimos en estudiantes que muestran escasa concentración y persistencia en el aprendizaje en línea, al ofrecerse módulos o unidades de alta densidad, muy extensos y de mucha duración, o actividades de aprendizaje escasamente motivadoras.

- Escasez de sistemas de apoyo y tutoría al estudiante que supongan motivación para la participación, la permanencia, soporte para la resolución de problemas académicos y psicológicos (Lozano-Díaz et al., 2020; Odriozola et al., 2020).

- Problemas al pasar de la imagen presencial del docente, de sus gestos y su voz, en directo, a una situación a distancia, en la que a veces todo se quedó en el frío texto.

- Cautelas, resistencias e, incluso, aversión de ciertos grupos docentes al uso de tecnologías.

- Falta de apoyos técnicos al profesorado presencial poco habituado a manejar estas tecnologías interactivas.

- No se combinaron adecuadamente las instancias de sesiones síncronas o asíncronas de presentaciones y orientaciones del docente, con el trabajo individual o de grupo de los estudiantes, síncrono o asíncrono.

- Se echó en falta un modelo más consolidado de evaluación formativa y continua, que hubiese reducido las grandes dificultades de una masiva evaluación final en línea, sobre todo en instituciones con grandes números de estudiantes.

- No se pudieron depurar los diferentes modelos de evaluación en línea que respondiesen a criterios de identificación fiable, calidad, igualdad, equidad, respeto a la privacidad, protección de datos, solidez de las infraestructuras tecnológicas, etc. 
Tras la cantidad de argumentos vertidos antes del confinamiento contra los sistemas universitarios no presenciales, que han venido siendo rebatidos una y otra vez a través de rigurosos estudios científicos, ahora, tras la pandemia, los que andan ansiosos de recuperar la presencialidad perdida aducen otros argumentos sobre lo negativo de hurtar al estudiante de su presencia en el campus físico.

Estamos seguros de que, en efecto, la experiencia de muchos docentes que se vieron obligados a digitalizar su acción educadora con motivo de la pandemia no ha sido positiva. Demasiado hicieron, de un día para otro, con convertirse de docentes presenciales a docentes en línea. Pero esa encomiable tarea obligada que hicieron nunca fue una enseñanza a distancia tal y como la venimos entendiendo desde hace algunas décadas. Se trató de una solución urgente, de ruptura, abrupta, de crisis, de emergencia, precipitada, sin diseño o, mejor, aplicando una pedagogía en línea o en remoto, basada en un diseño pedagógico para la presencia. En muchos casos, se trató de volcar lo que se venía haciendo en el aula presencial, fue una enseñanza de emergencia en remoto (Hodges et al., 2020) o, mejor, educación de emergencia en remoto, así quedarían incluidos conceptos como enseñanza, instrucción, aprendizaje. Nada tuvo que ver esto con lo que hubiese sido una educación a distancia digital bien diseñada e implementada (Hodges et al., 2020). Además de que no se tuvo en cuenta el factor de fatiga referida a la réplica síncrona, a través de la pantalla, de una clase presencial, "fatiga Zoom" (Wiederhold, 2020).

Naturalmente habrá quienes sacarán conclusiones de esta situación referidas a las debilidades de la educación a distancia, sin detenerse a pensar:

- que no hubo planificación,

- que existieron problemas de conectividad y de equipamiento para muchos afectados,

- que no se contó con plan alguno de capacitación docente,

- ni de preparación de los estudiantes respecto a la autodisciplina y autorregulación de su trabajo, más necesarios en estos entornos,

- y que la actitud negativa de muchos docentes, podrían desnivelar los datos finales de cualquier valoración.

Aun así, habrá muchos resultados positivos, gracias a la entrega y espíritu innovador de otros docentes.

Si nos trasladamos a las épocas más duras del confinamiento, hubo muchos docentes que abominaron, más que antes, de las pedagogías no presenciales, convenciéndose de que tenían razón cuando negaban posibilidades educativas dignas de consideración a esas modalidades digitales. Naturalmente, se trataba de docentes que a fuerza del mandato de las autoridades sanitarias tuvieron que virar $180^{\circ}$ en sus formas de hacer educación. Probablemente se limitaron a replicar su acción pedagógica en formato digital, fuese síncrono o asíncrono. Y, casi seguro, no funcionó. 
Pero con seguridad, en sentido frontalmente contrario, hubo docentes que se convirtieron de forma radical, al estilo de como lo hizo cayendo del caballo, San Pablo. Pero este santo parece que contó con el impulso divino que quizás no se prodigue en el tema que nos ocupa. Algunos de estos "conversos" han llegado a ser prolijos en "vender" modelos y metodologías maravillosas, alejadas de la relación presencial entre docente y estudiante. De ignorar, o incluso atacar, a las metodologías a distancia, sin estudio ni reflexión ni asesoramiento previos, escribían, argumentaban y defendían el reciente "hallazgo".

Otros fueron descubriendo una serie de valores, antes ignorados, de las tecnologías aplicadas a la educación. No como para abjurar de la enseñanza presencial que siempre hicieron bien, pero sí para valorar otras posibilidades que podrían enriquecer sus experiencias docentes futuras, a través de algunas propuestas combinadas o de incipiente hibridación metodológica. Posiblemente se pusieron a estudiar, a debatir, a asesorarse, etc.

No faltó aquel profesorado que, aún desenvolviéndose en entornos básicamente presenciales, ya habían experimentado y descubierto las bondades de ciertas tecnologías aplicadas a determinadas acciones educativas, en coherencia con algunos objetivos y competencias establecidos y en función de las exigencias de las actividades de aprendizaje propuestas. Estos tuvieron mínimo problema ante el obligado cambio de contexto.

Naturalmente, ya estaban aquellos que se desenvolvían en contextos en línea, para quienes el confinamiento, a efectos educativos, no tuvo especiales dificultades. Y finalmente, quienes, además de conocer la práctica educativa en estos entornos, se dedicaron desde años a estudiar, investigar, innovar y escribir sobre este fenómeno educativo que, manejado con rigor, vienen aportando soluciones de valor dentro del ámbito educativo.

Por otra parte, la situación del confinamiento se convirtió en una gran oportunidad de mercado, tanto para todos aquellos nuevos "conversos" que, quizás, pretendieron vender sus nuevos postulados teóricos y técnicos de respuesta a la pandemia y, sobre todo, para empresas tecnológicas que ofrecieron sus productos, generalmente plataformas para el aprendizaje digital. Plataformas no siempre respaldadas por la debida verificación técnica, ni por sólidos principios pedagógicos (Hodges et al., 2020).

En todo caso, esta educación remota de emergencia que permitió a muchos estudiantes continuar sus estudios en medio de la pandemia, no es una solución válida a largo plazo. De cara al futuro, las instituciones deben desarrollar planes educativos sostenibles que puedan resistir los desafíos y las incógnitas de este u otros escenarios similares que pudieran acaecer (Johnson, Veletsianos y Seaman, 2020), sea para tiempos de confinamiento total o parcial, continuado o intermitente, de forma que no se generen situaciones tan traumáticas como las vividas en 2020. 


\section{POSCONFINAMIENTO}

Diferentes administraciones públicas e instituciones educativas han propuesto, vienen planificando, o se verán obligadas a hacerlo, una etapa educativa posconfinamiento, con diferentes escenarios ante las incertidumbres futuras con respecto a la evolución de la pandemia, de ésta o de otras venideras. Porque, incluso si no se levanta el confinamiento en algunos países, como en el peor de los contextos, o si existe una vuelta atrás, a un nuevo confinamiento sea total o parcial, de larga o corta duración, las cosas han de hacerse de otra manera. Y esto, sea en tiempos de COVID-19 o en previsión de otra nueva pandemia.

$\mathrm{Al}$ menos en los primeros meses del posconfinamiento necesariamente se producirá un tránsito desde las actividades educativas tradicionales, centradas en el docente y, generalmente, basadas en exposiciones magistrales tanto presenciales (preconfinamiento) como online (confinamiento), hacia acciones más centradas en el estudiante, incluidas actividades en grupo, discusiones, actividades de aprendizaje práctico y un uso más limitado de exposiciones por parte de los docentes (Zhu y Liu, 2020). Obviamente, esto requiere un replanteamiento conceptual y filosófico de la naturaleza de la enseñanza y el aprendizaje y de los roles y conexiones entre el profesorado, los alumnos y los materiales didácticos (Jandrić et al., 2018).

Además de la necesaria flexibilización, reestructuración y adecuación de espacios educativos físicos que preserven la habitabilidad y seguridad sanitaria y que acojan servicios tecnológicos, se hacen precisos nuevos enfoques pedagógicos más abiertos, diversos, combinados y flexibles, para abordar una situación que en el ámbito sanitario aún no fue superada. Estaríamos hablando de experiencias de blendedlearning, ya antiguas (Procter, 2003), aunque algunos las descubrieron ahora, pero también de escenarios de presencialidad discontinua o variable, según manden las circunstancias sanitarias.

En todo caso, más allá del rediseño de aulas, la adaptación de nuevos espacios multiusos o al aire libre y de la distribución de grupos de alumnos más reducidos, no será posible atender a todos en formato presencial durante las mismas horas y espacios que se utilizaban en tiempos preCOVID. No existen los recursos, ni el personal docente suficiente, ni los espacios necesarios para hacerlo con grupos pequeños. Se pueden arbitrar soluciones provisionales si el empeño sigue siendo todos en presencia durante todo el horario lectivo. Pero hay que tener previstas situaciones de enseñanza-aprendizaje combinados, mixtos, híbridos. E, incluso, prever otro escenario de vuelta al confinamiento por el recrudecimiento de esta o de otra pandemia.

Los sistemas presenciales tendrán la oportunidad de evaluar con rigor y objetividad lo que se ganó y lo que se perdió en los tiempos de confinamiento total, así como las diferencias en la calidad de los aprendizajes adquiridos según zonas geográficas, instituciones, docentes, metodologías, sincronía, asincronía, etc. No se sabe durante cuánto tiempo, pero habrá que convivir con estas nuevas formas 
de enseñar y aprender. Y es muy probable que la "normalidad pedagógica" en la universidad llegue a establecerse aprovechando lo mucho aprendido durante estos tiempos de crisis sanitaria, relacionado con modelos combinados o híbridos.

La gran ventaja, con respecto a los tiempos de confinamiento, es la de que hemos aprendido y vamos teniendo tiempo para diseñar esos nuevos enfoques pedagógicos de los meses futuros, sin olvidar lo que pasó en los tiempos de cierre físico de los centros, en los que algunos estudiantes en desventaja perdieron aprendizajes que deberán recuperar, por lo que será responsabilidad de las administraciones y, más concretamente de los centros y universidades la de establecer los instrumentos que se consideren eficaces para cubrir esas pérdidas y acercarse a la equidad. Una vez supuesto ese equilibrio académico de todos los estudiantes, han de arbitrarse esos nuevos modelos híbridos a los que venimos aludiendo.

Si ya se realizó la valoración de aquello que se perdió y de cuánto se ganó en tiempos educativos de confinamiento, es el momento. Ya esos docentes llevaban décadas de práctica educativa presencial. Ahora, la emergencia sanitaria les obligó a otras prácticas totalmente inusuales para ellos. Será la oportunidad de detectar las debilidades de la enseñanza presencial que podrían compensarse con determinadas fortalezas de la educación a distancia digital. ¿Cuánto de las más groseras debilidades de la educación a distancia pueden fortalecerse con bondades evidentes de la formación presencial? Sería ocasión de aprender de los aciertos y errores de uno y otro modelo.

Aunque, insistimos, es importante a la hora de esta evaluación, el hacer la salvedad de que la mayoría de los docentes presenciales, diseñaron para la presencia y desarrollaron su práctica docente siempre en presencia, se supone que mejorándola año a año. Y que esos mismos docentes se vieron abruptamente empujados a practicar, sin diseño ni preparación, en una docencia a distancia. No cabe duda de que en las valoraciones que las instituciones estén realizando sobre estas percepciones personales, habrán de aislar esas variables, con el fin de ser objetivos a la hora de decidir hasta dónde desean llegar, hasta dónde profundizar esos nuevos enfoques pedagógicos.

Con esos elementos positivos (fortalezas) y negativos (debilidades) de uno y otro modelo habrá de armarse ese nuevo enfoque, manteniendo ese principio con anterioridad esbozado de que nadie se quede atrás (equidad) por razones de desventaja socioeconómica. Será necesario que las administraciones públicas regulen estos nuevos modelos educativos. En todo caso, ¿qué soluciones de hibridación, combinación e integración podrán implementarse o se están anunciando ya, en función de los resultados de esas evaluaciones referidas? (Stein y Graham, 2020).

Con la perentoria necesidad de reorganizar el sistema educativo tras la pandemia, vienen surgiendo numerosas variantes y propuestas. Pero todas ellas se desenvuelven dentro del continuum presencia-distancia (en línea), (onsiteonline). ¿Qué porcentaje de una y otra? Mirando las dos gráficas de la figura 3, podría concluirse que las soluciones son múltiples y muy diferentes. 
Figura 3. Posibilidades educativas dentro del continuum presencia-distancia

A)

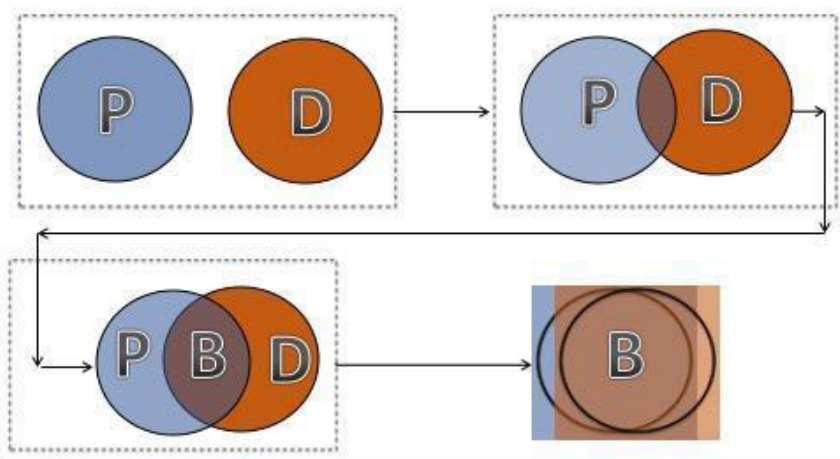

B)

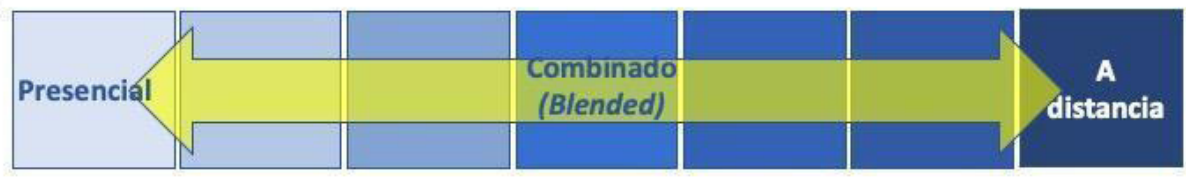

Podríamos encontrarnos desde situaciones de sincronía en remoto, propias del confinamiento, hasta las previsiones posconfinamiento, de los sistemas rotatorios, que garantizan mejor la distancia de seguridad sanitaria y diluyen la masificación, y que parece que en esta época se están imponiendo. Realicemos una aproximación de posibles propuestas para estos tiempos, o para indeseadas futuras emergencias similares. Estas serían algunas posibilidades (a y b, para tiempos de confinamiento y las restantes para tiempos pos-COVID).

a. Sincronía en remoto (10o\%). En estos tiempos de pandemia y confinamiento se vino imponiendo en muchos centros un modelo que convirtió la sincronía presencial en una sincronía en remoto. Se trataba de replicar, sin más, toda la actividad del docente y estudiantes en el aula, mediante una conexión síncrona, en muchos casos respetando, incluso, la misma distribución y horarios de la época preCOVID.

b. Sincronía y asincronía. (También para tiempos de confinamiento). En formato síncrono, por ejemplo, las exposiciones y orientaciones del docente, para realizar el restante tiempo de trabajo también en línea, pero en formato asíncrono. La hibridación o mezcla sería entre lo síncrono y lo asíncrono, más que entre la presencia (que no se produjo) y la distancia.

c. Apuesta por la presencialidad total. (Posconfinamiento). En estos casos al primar la seguridad sanitaria se hará preciso, por la necesidad de guardar las preceptivas distancias físicas, multiplicar y adaptar los espacios, así como 
aumentar sustancialmente el número de docentes. Es opción extendida en bastantes países, generalmente para los niveles inferiores del sistema educativo, pero su viabilidad se antoja algo complicada para ser cumplida $100 \%$ durante un tiempo relativamente prolongado, dada la escasez de recursos, sobre todo en determinados países.

d. Por mitades, tercios..., alternos. (Posconfinamiento). Si nos referimos a los tiempos presenciales, la mitad de la clase o grupo de alumnos asiste por la mañana y la otra mitad por la tarde. Una mitad sería a distancia y según decisión, con formato síncrono o asíncrono. Esas rotaciones o alternancias pueden darse, en lugar de mañana y tarde, en días alternos de la semana, dividiendo el $5^{\circ}$ día por la mitad, o haciendo ese $5^{\circ}$ día de la semana en formato virtual para todos. Pueden rotar el 50\% de los estudiantes, o un tercio cada vez, o el 25\%, etc., según las necesidades y disposición de espacio físico. Las combinaciones son múltiples, rotación por semanas, por quincenas, meses, alternando el cuatrimestre/ semestre, con diferente proporcionalidad presencia/distancia, etc. El formato síncrono podría ser:

- síncrono/virtual para todos; o

- hibridación síncrona o concurrencia/simultaneidad de aulas, que supondría que mientras la mitad (o tercio) de los estudiantes siguen en presencia (aula presencial) las orientaciones del docente y dinámica del grupo, la otra mitad (o dos tercios) sigue esas mismas orientaciones y participa de esa dinámica, desde sus hogares en conexión síncrona (aula virtual) (Beatty, 2019), o mediante el sistema de "aulas espejo" que se podrían establecer en locales, propios o no, de la misma institución. En ambas situaciones, la conexión sería en directo siguiendo la misma dinámica del grupo que asiste presencialmente;

- opcionalidad. Asisten a más sesiones presenciales aquellos que tienen problemas de brecha digital (de conectividad o de acceso con dispositivos), o determinadas dificultades de aprendizaje, problemas de conciliación familiar o, sencillamente, los que quieren asisten, previa comunicación al centro, y los que no, se quedan en casa (¿auto-blended?).

e. Aula invertida. (Posconfinamiento). Si se opta por el formato asíncrono de esa mitad que trabajaría desde casa, podría tratarse de un modelo de aula invertida que se repetiría con cada grupo de forma idéntica y alterna, o con todos a la vez.

f. HyFlex. (Posconfinamiento). Si incluimos posibilidades máximas de flexibilidad, podríamos hablar de las tipologías HyFlex (Hybrid + Flexible) que sería un modelo combinado o híbrido pero adaptado al máximo a las posibilidades del estudiante. Es decir, el estudiante es más protagonista en la toma de decisiones en cuanto a su proceso de aprendizaje, puede decidir, por 
ejemplo, qué actividades en presencia o en línea, síncronas o asíncronas, qué porcentaje de sesiones en una u otra modalidad... (Beatty, 2007; Miller, Risser y Griffiths, 2013).

g. Modelo de lista de reproducción. (Posconfinamiento). Muy similar al anterior modelo citado. Se trataría de una especie de rotación individual. Los docentes ofrecen a los estudiantes una secuencia de actividades de aprendizaje relacionadas con una unidad de estudio, que éstos podrán ir acometiendo a su ritmo. Esas actividades deberán ser de lo más variadas posible, individuales o grupales (Tucker, 2020). El docente podrá atender a estudiantes individual o grupalmente, según se precise.

Algunas consideraciones que habrían de tenerse en cuenta en determinados escenarios de los apuntados:

a. Porcentajes. Tiempo de presencia/distancia. 75-25\%; 50-50\%; 25-75\%, de cada alumno, de cada profesor.

b. Proporción docente/estudiantes. Resulta evidente que en gran parte de estas consideraciones se hacen precisos refuerzos docentes. Deberá establecerse una nueva ratio, muy diferente a la existente en la etapa preCOVID.

c. Tipo de presencia en línea del docente. Niveles de presencia activa del docente, en vídeo, audio, foros, chat, etc. Tiempos de dedicación a las diferentes tareas docentes (carga y saturación docente).

d. Proporción de sincronía/asincronía en los tiempos en línea. Videollamadas grupales, individuales, presentaciones, debates, etc.

e. Disponibilidad de conectividad y recursos por parte de la institución y de los estudiantes de ese centro, arbitrando modelos híbridos flexibles y adaptados a esas necesidades de los grupos más vulnerables.

f. Hibridación relativa a las prácticas. Podría desarrollarse un sistema híbrido o combinado que contemple la realización de las prácticas y de otras actividades específicas en formato presencial y el resto a través de los sistemas digitales, dividiendo en grupos más pequeños. O bien, proponer en general, contenidos teóricos online y contenidos prácticos en presencia.

Respecto a este último punto habrá de aclararse que las tecnologías están permitiendo hoy inmensas posibilidades para la realización de diversidad de prácticas en entornos simulados y laboratorios remotos, gracias a la inteligencia artificial, la realidad aumentada, realidad virtual, extendida, el big data, machine learning, etc.

Damos por supuesto que las soluciones propuestas, y otras que pudieran implementarse, tienen muy diferente concreción en el sistema no universitario. Ahí se agudizarán los problemas de las desigualdades socioeconómicas correlativas con la brecha digital y problemas de conciliación familiar o de tipo laboral de los padres con respecto a los niños que se tuvieran que quedar en casa. En la universidad 
pueden reducirse e incluso anularse estas dificultades. Las ideas vertidas en este trabajo tienen como fundamental objetivo la educación superior.

\section{RECOPILANDO. LA FLEXIBILIDAD}

En un monográfico de esta misma revista RIED del año 2018, Vol. 21(1), dedicado al blended-learning, en su artículo editorial se defendía el concepto de aprendizaje integrado. Se trataría con este concepto, no de buscar puntos intermedios, ni intersecciones entre los modelos presenciales y a distancia, sino de integrar, combinar, compensar, armonizar, complementar, enriquecer, conjugar e integrar, los medios, recursos, tecnologías, las comunicaciones, las metodologías, actividades, estrategias y técnicas..., sean presenciales o a distancia, sean síncronas o asíncronas, más apropiados para satisfacer cada necesidad concreta de aprendizaje en cada momento o situación (también en tiempos de pandemia o pospandemia), tratando de encontrar el mejor equilibrio posible entre tales variables curriculares. Bien es sabido que la tecnología por sí mismo no es garante de éxito educativo (Reich, 2020). Se trataría de una flexibilidad ampliamente considerada. Remitimos a dicho artículo.

Probablemente el equilibrio de todos esos elementos, combinándolos con la flexibilidad adecuada, podría dar respuesta a las diferentes necesidades de formación, especialmente en tiempos de posCOVID. Una vez que exista la decisión de adoptar un determinado modelo, sea para situaciones de confinamiento total (educación a distancia digital 100\%) o para tiempos posteriores (educación híbrida o combinada) y, más allá de las medidas de prevención sanitaria (uso de mascarillas, higiene, distancia de seguridad, tamaño de los grupos, uso de zonas comunes, etc.), habrán de considerarse, entre otras posibles, las siguientes variables de carácter más pedagógico, priorizando el concepto de flexibilidad:

- el impulso decidido a la digitalización de los centros que posibilite mayor grado de flexibilidad;

- las posibilidades de flexibilidad que ofrece el ordenamiento educativo del país o de las comunidades autónomas y regiones, probablemente adaptado también a las circunstancias;

- los escenarios permitidos de flexibilidad de la organización docente ante situaciones de posconfinamiento que impidan la presencialidad $100 \%$ o de confinamientos intermitentes, con el fin de alternar modalidades educativas, mediante un desarrollo curricular híbrido, mixto, combinado o de discontinuidad presencia-distancia;

- flexibilidad con los recursos, sean internos o externos, que se precisarán para acometer el cambio;

- flexibilidad de los tiempos, cronograma, necesarios para el inicio del modelo, para el diseño y adaptación de contenidos, actividades y líneas de interacción; 
- flexibilidad para elaborar o seleccionar materiales de audio, vídeo, imágenes, textos, etc.;

- flexibilidad para prevenir o proveer nuevas instancias de evaluación garantistas de la identidad, la calidad, la igualdad, equidad, solidez de los sistemas tecnológicos, respeto a la privacidad...;

- flexibilidad para la readaptación de espacios físicos y dotaciones tecnológicas para los grupos presenciales.

Otras cuestiones que habrán de ser tenidas en cuenta:

- los estados de ánimo, impacto socioemocional y percepciones de los docentes, personal de administración y servicios, estudiantes y familias tras las experiencias durante el confinamiento;

- la necesidad de recuperar aprendizajes por parte de aquellos estudiantes más perjudicados por la imposibilidad de un aprendizaje presencial;

- la previsión de formación del alumnado en las necesarias competencias digitales y en la prevención de adicciones y mal uso de las tecnologías;

- las soluciones posibles para aquellos estudiantes más vulnerables, que sufran en sentido negativo el impacto de las desigualdades sociales y brecha digital;

- la citada fatiga de una continuada exposición a la pantalla por parte de los estudiantes;

- la capacidad de respuesta de las infraestructuras tecnológicas;

- la consideración de los problemas de privacidad, confidencialidad y protección de datos ante las tecnologías invasivas;

- dificultades económicas de mayor dotación de profesorado que exige la reducción de alumnos por grupos;

- las necesidades de formación específica del profesorado en competencias metodológicas para la docencia en línea y en competencias digitales que reduzca la brecha digital generacional;

- la disposición y adecuación de las unidades o centros de apoyo técnico al profesorado;

- las investigaciones más relevantes de corte cualitativo y cuantitativo que hayan podido realizarse con el fin de valorar los modelos con más garantías de éxito;

- a nivel local e institucional, valorar cuáles fueron las debilidades y fortalezas más notables durante la época de confinamiento y educación a distancia $100 \%$, considerando las cautelas ya señaladas en este trabajo;

- potenciar los niveles de cooperación en redes docentes, institucionales y organizacionales, públicas y privadas, que propicien la cooperación para la búsqueda de los mejores modelos, las mejores prácticas y el software más apropiado. 
Ya se ha escrito bastante al respecto, la pandemia puede generar de forma indirecta algunas ventajas, algunos beneficios a la sociedad. Sin duda, elementos para la reflexión en educación, sí que ha suscitado, de tal modo que postulados como los indicados más arriba, en este momento ya no son tan discutidos, ni observados con tantas reservas o desconfianza.

Ciertamente que, durante el confinamiento, en los niveles no universitarios se aportaron soluciones muy provisionales que, aunque dejarán elementos de reflexión para adoptar determinadas innovaciones, finalizarán gran parte de ellas una vez superada la crisis. Sin embargo, en la universidad probablemente será diferente. Las modalidades a distancia, digitales, en línea y flexibles van a ser aprovechadas de forma muy generalizada una vez superada la pandemia.

Que existen instituciones y docentes que están deseando volver al tradicional modelo presencial, por supuesto. Pero ¿̇podrá dudarse que, incluso entre éstos que anhelan la presencialidad $100 \%$, en el futuro sus prácticas educativas se van a ver moduladas y mucho más enriquecidas, mediadas o complementadas, por las tecnologías digitales?

Por otra parte, esos mismos docentes deberán ser conscientes de que igual la realidad empuja a tener que adoptar forzosamente modelos híbridos o, quién sabe si, de nuevo, a distancia $100 \%$ por regreso a un nuevo confinamiento. Y ese regreso habría de hacerse de forma muy diferente a como se hizo en esta ocasión. De ahí que esos escenarios deberían estar previstos en las programaciones de los centros realizando diseños convertibles, reversibles y adaptados a las nuevas situaciones, sin que sufra en exceso el desarrollo curricular. Por eso la formación de docentes para estas situaciones singulares o de emergencia se impone y debería ser objetivo prioritario. Igual habrían de prepararse a los estudiantes por si se diesen situaciones diferentes a las inicialmente previstas y hubiese que migrar de nuevo a entornos en línea. $\mathrm{Y}$ en los niveles no universitarios, también habría que concienciar a las familias.

El impacto de esta pandemia y la concomitante crisis económica, han generado un cambio en cómo, cuándo y dónde ocurre el aprendizaje del estudiante (Fox, et al., 2020). La renovación e innovación pedagógica siempre recomendada y, generalmente, aplazada, podrá contar ahora con la gran oportunidad para hacerse realidad y ganar en calidad y equidad educativas (Pedró, 2020). Se precisan para un futuro inmediato sistemas educativos resilientes, con capacidad de respuesta ante situaciones de emergencia y con salvaguarda para aminorar las desigualdades que se vieron agravadas como nunca. En realidad, el COVID-19 puede presentarse como acelerador de la transformación de la educación superior que supondrá que el aprendizaje en línea y flexible vinieron para quedarse (Naffi, 2020).

Bien se sabe que una variable curricular esencial de todo proceso educativo es la evaluación. Sobre ella se estableció durante el confinamiento un gran debate que aún pervive, sobre fórmulas más adecuadas para llevar a cabo estrategias y técnicas de evaluación, habida cuenta de los problemas actuales para implementar 
una evaluación universal de carácter presencial. Invitamos al próximo monográfico de esta revista RIED que, precisamente, versará sobre la evaluación digital y la digitalización de la evaluación.

No quisiéramos terminar sin animar para abordar investigación científica sobre muchas de las reflexiones aquí apuntadas. La situación fue de emergencia, las soluciones también lo están siendo. De ahí la necesidad de respaldo científico para la cantidad de propuestas que se vienen haciendo, con el fin de consolidar sólo aquellas que ofrecen resultados de calidad contrastada.

\section{NOTAS}

1. Mapas actualizados sobre el impacto del COVID-19 en la Educación. En agosto de 2020, existían cerca de 1.500 millones de estudiantes afectados por el cierre de centros educativos, que suponen el $70 \%$ de los estudiantes matriculados y 160 países con cierres totales o parciales. Ver mapas: https://es.unesco.org/covid19/ educationresponse y https://www.worldbank.org/en/data/interactive/2020/03/24/ world-bank-education-and-covid-19

\section{REFERENCIAS}

Bao, W. (2020). COVID-19 y la enseñanza en línea en la educación superior: un estudio de caso de la Universidad de Pekín. Human Behavior and Emerging Technologies, 2(2), 113-115. https://doi. org/10.1002/hbe2.191

Beatty, B. (2007). Transitioning to an Online World: Using HyFlex Courses to Bridge the Gap. In C. Montgomerie y J. Seale (Eds.), Proceedings of ED-MEDIA 2007--World Conference on Educational Multimedia, Hypermedia \& Telecommunications (pp. 2701-2706). Vancouver. https://www. learntechlib.org/primary/p/25752/

Beatty, B. J. (2019). Hybrid-Flexible Course Design. Implementing studentdirected hybrid classes. Provo, Utah: EdTech Books.

Dreesen, T., Akseer, S., Brossard, M., Dewan, P., Giraldo, J. P., Kamei, A., Mizunoya, S., y Ortiz, S. (2020). Promising practices for equitable remote learning Emerging lessons from COVID-19 education responses in 127 countries. Innocenti
Research Brief. UNICEF. https://cutt.ly/ ufffokG

Fox, K., Bryant, G., Lin, N., y Srinivasa, N. (2020). Time for Class - COVID-19 Edition Part 1: A National Survey of Faculty during COVID-19. Tyton Partners and Every Learner Everywhere, July 8, 32 pp. https://www.everylearnereverywhere. org/resources/time-for-class-covid-19edition/

Hart, J. (2020). Top 200 Tools for Learning 2O2O, https://www.toptools4learning. com/

Hodges, C., Moore, S., Lockee, B., Trust, T., y Bond, A. (2020). La diferencia entre la enseñanza remota de emergencia y el aprendizaje en línea. Educause Review. https://er.educause.edu/ articles $/ 2020 / 3 /$ the-difference-betweenemergency-remote-teaching-and-onlinelearning

IESALC-UNESCO (2020). El coronavirus-19 y la educación superior: impacto $y$ recomendaciones. https://cutt.ly/ xdHJuhK 
Jandrić P., Knox J., Besley T., Ryberg T., Suoranta J., y Hayes S. (2018). Postdigital science and education. Educational Philosophy and Theory, 50(10): 893899. https://doi.org/10.1080/00131857. 2018.1454000

Johnson, N., Veletsianos, G., y Seaman, J. (2020). U.S. Faculty and Administrators' Experiences and Approaches in the Early Weeks of the COVID-19 Pandemic. Online Learning, 24(2). https://doi. org/10.24059/olj.v24i2.2285

Lozano-Díaz, A., Fernández-Prados, J. S., Figueredo Canosa,V.,y Martínez Martínez, A. M. (2020). Impactos del confinamiento por el COVID-19 entre universitarios: Satisfacción Vital, Resiliencia y Capital Social Online, International Journal of Sociology of Education, Special Issue: COVID-19 Crisis and Socioeducative Inequalities and Strategies to Overcome them, 79-104. https://doi.org/10.17583/ rise.2020.5925

Lozano, J. C., Cooper, E., y Soto, A. (2020). 5 Trends In VR Training Solutions To Elevate Your Blended Learning Program. eLearning Industry. https://cutt.ly/ lfrpCqj

Miller, J., Risser, M., Griffiths, R. (2013). Student Choice, Instructor Flexibility: Moving Beyond the Blended Instructional Model. Issues and Trends in Educational Technology, 1(1), 8-24. University of Arizona Libraries. https://www. learntechlib.org/p/129818/

Naffi, N.(2020). Disruption in and by Centres for Teaching and Learning During the COVID-19 Pandemic: Leading the Future of Higher: L'Observatoire Internationale sur les Impacts Sociétaux de l'IA et du Numerique and the Government of Québec, https://cutt.ly/6fQZibh

Odriozola, P., Planchuelo, A., Irurtia, M. J., de Luis, R. (2020). Psychological effects of the COVID-19 outbreak and lockdown among students and workers of a Spanish university. Psychiatry
Research, 290. https://doi.org/10.1016/j. psychres.2020.113108

Pedró, F. (2020). COVID-19 y educación superior en América Latina y el Caribe: efectos, impactos y recomendaciones políticas. Análisis Carolina, https://cutt. ly/sfytfUZ. https://doi.org/10.33960/ AC 36.2020

Procter, C. T. (2003). Blended learning in practice. Education in a Changing Environment conference, (September 2003) Salford. http://usir.salford.ac.uk/ id/eprint/27428/

Research and Markets (2019). Online Education Market \& Global Forecast, by End User, Learning Mode (Self-Paced, Instructor Led), Technology, Country, Company. https://cutt.ly/QdHHPcI

Reich, J. (2020). Failure to Disrupt. Why Technology Alone Can't Transform Education. Harvard University Press.

Singh V., y Thurman A. (2019). How many ways can we define online learning? A systematic literature review of definitions of online learning (1988-2018). American Journal of Distance Education, 33(4), 289-306. https://doi.org/10.1080/0892 3647.2019 .1663082

Stein, J., y Graham, C. R. (2020). Essentials for blended learning: A standardsbased guide. Routledge. https://doi. org/10.4324/9781351043991

Tucker, C. (2020). The Concurrent Classroom: Using Blended Learning Models to Teach Students In-person and Online Simultaneously. @Catlin Tucker. https://catlintucker.com/2020/09/ concurrent-classroom-blended-learningmodels/

u-Multirank (2020). About 60\% of universities reported online learning provisions in their strategic planning preCOVID-19, but only few appeared to be prepared for a quick shift to full online programmes. https://cutt.ly/VfGDArk 
UN (2020a). Policy Brief: Education during COVID-19 and beyond (August 2020). United Nations. https://cutt.ly/bdHJEhX

UN (2020b). Policy Brief: The World of Work and COVID-19 (June, 2020). United Nations. https://cutt.ly/6fpDKHF UNESCO (2020). Startling digital divides in distance learning emerge. https://cutt. ly/adH2SRS.

Wiederhold,B.K.(2020).ConnectingThrough Technology During the Coronavirus Disease 2019 Pandemic: Avoiding "Zoom Fatigue". Cyberpsychology, Behavior, and Social Networking, 23(7). https:// doi.org/10.1089/cyber.2020.29188.bkw
World Bank (2020). How countries are using edtech (including online learning, radio, television, texting) to support access to remote learning during the COVID-19 pandemic. World Bank. https://cutt.ly/ ZfuND7b.

World Economic Forum, (2020). 3 ways the coronavirus pandemic could reshape education. https://url2.cl/N3klw.

Zhu, X., y Liu, J. (2020). Education in and After Covid-19: Immediate Responses and Long-Term Visions. Postdigital Science and Education, 1-5. https://doi. org/10.1007/s42438-020-00126-3 